\title{
QRQueijo: Sistema de Emissão e Validação de Identificadores de Queijos
}

\section{Utilizando Blockchain}

\section{QRQueijo: Emission and Validation System of Cheese Identifiers Using Blockchain}

Anderson Melo de Morais*1, Ademário José da Silva ${ }^{1}$, Sérgio Francisco Tavares de Oliveira Mendonça ${ }^{2}$

${ }^{1}$ Universidade Federal Rural de Pernambuco, Pernambuco, Brasil.

${ }^{2}$ Universidade Federal do Agreste de Pernambuco, Pernambuco, Brasil.

\begin{tabular}{|c|c|}
\hline I NFO ARTIGO & RES U MO \\
\hline $\begin{array}{l}\text { Palavras-chave: } \\
\text { Blockchain, } \\
\text { Segurança, } \\
\text { Rastreabilidade. }\end{array}$ & $\begin{array}{l}\text { Este trabalho teve como objetivo desenvolver uma aplicação que possa gerar } \\
\text { identificadores para queijos e por meio de uma rede Blockchain validar tais } \\
\text { informações. Para isso realizou-se um estudo sobre o funcionamento de uma } \\
\text { Blockchain e suas características de segurança, imutabilidade e } \\
\text { escalabilidade. Identificou-se a possibilidade de uso de Blockchain na } \\
\text { rastreabilidade de produtos alimentícios. Com isso, realizou-se a modelagem } \\
\text { e implementação de um sistema para registro de dados sobre queijos e seus } \\
\text { fabricantes em uma Blockchain. Tal registro permite validar as informações e } \\
\text { as disponibilizar aos consumidores por meio do aplicativo móvel QRQuejio e } \\
\text { averiguar a autenticidade dos dados, por meio de uma leitura na base de dados } \\
\text { do sistema e na Blockchain, confirmando assim a autenticidade dos registros. }\end{array}$ \\
\hline ARTICLE INFO & A B S T R ACT \\
\hline $\begin{array}{l}\text { Keywords: } \\
\text { Blockchain, } \\
\text { Safety, } \\
\text { Traceability. }\end{array}$ & $\begin{array}{l}\text { This work aimed to develop an application that can generate identifiers for } \\
\text { cheese and through a Blockchain network to validate such information. For } \\
\text { this, a study was carried out on the functioning of a Blockchain and its } \\
\text { characteristics of security, immutability, and scalability. The possibility of } \\
\text { using Blockchain in the traceability of food products was identified. With this, } \\
\text { the modeling and implementation of a system for recording data about cheeses } \\
\text { and their manufacturers in a Blockchain were carried out. Such registration } \\
\text { allows us to validate the information and make it available to consumers } \\
\text { through the QRQuejio mobile application and to verify the authenticity of the } \\
\text { data, through reading in the system database and the Blockchain, thus } \\
\text { confirming the authenticity of the records }\end{array}$ \\
\hline
\end{tabular}

\section{Introdução}

As tecnologias estão cada vez mais presentes na vida das pessoas, inseridas em ambientes domésticos, sociais, acadêmicos, profissionais e industriais. Os investimentos no setor de Tecnologia da Informação (TI) atingiram US\$ 3,76 trilhões em 2019, com um aumento de mais de 3,2\% em relação a 2018 (COSTELLO e OMALE, 2019). A rápida evolução tecnológica implica em um aumento da disponibilidade e alcance das informações, consequência do grande consumo desses recursos pela população. A informação tem um grande valor para as tomadas de decisões e são de fundamental importância para o desenvolvimento das empresas e instituições (MORESI, 2000). A preocupação com a segurança e a integridade dessas informações são fatores primordiais para

\footnotetext{
* Correspondência para autor:

anderson.ufrpe@gmail.com (de Morais, A.M.) (ORCID: 0000-0001-7795-7183), ademariocc@gmail.com (da Silva, A.J.), sftdom@gmail.com (Mendonça, S.F.T.O.).
}

1679-1827 (C) 2019 Gest@o.org. 
todos que às mantém

Atualmente a maior parte das informações são geradas e processadas através de sistemas distribuídos, utilizados para atender as diversas necessidades dos usuários. Esses sistemas são definidos como sendo uma coleção de computadores independentes entre si, que se apresenta ao usuário como um sistema único e coerente (TANENBAUM, 2007). Garantir a segurança de tais sistemas é um fator fundamental em qualquer contexto computacional. Portanto, três fatos são considerados essenciais para a seguridade das informações: a confiabilidade, a disponibilidade e a integridade (SCARFONE, 2008).

As redes Blockchain apresentam-se como uma tecnologia que assegura tais fatores de segurança, pois, visa a descentralização como aspecto principal (NAKAMOTO, 2008). Consistem em bases de dados distribuídas, que armazenam todas as transações que ocorrem entre os usuários da rede (MOUGAYAR, 2018). Cada computador na rede, chamado nó, guarda uma cópia da cadeia de registros. As informações são armazenadas em blocos e listados de forma encadeada. Cada bloco possui suas informações criptografadas e mantém uma referência para o bloco anterior. É essa característica da rede que impede que novos blocos sejam inseridos ou modificados, sem que tal alteração seja imediatamente percebida por todos os demais nós da cadeia.

Este trabalho tem como objetivo a implementação de um sistema de rastreamento de queijos voltado aos produtores regionais do estado de Pernambuco, baseado em Blockchain, que permitirá o registro de toda a sua cadeia de produção de forma segura e inalterável (NAKAMOTO, 2008), diminuindo assim os riscos de fraudes por meio dos fornecedores e fabricantes. Buscou-se desenvolver também um aplicativo móvel, intitulado QRQueijo, para que os consumidores possam rastrear os produtos e verificar aspectos como: validade, data de fabricação, nome e endereço do fabricante, bem como o percurso do queijo até a sua mesa.

Realizou-se um estudo acerca do tema, motivado a partir da experiência de consumidores de queijos regionais, notou-se que eles não possuíam informações sobre suas características e locais de produção. A falta de tais informações pode gerar desconfianças e falta de garantias aos consumidores. Fato este comprovado por notícias como "a destruição de 13 mil queijos de leite cru da Serra da Canastra por terem sidos mantidos em estoque e comercializados sem registro em órgão fiscalizador e sem identificação de origem (MOL, 2015). Isso tem levantado discussões sobre a rastreabilidade dos queijos, desde a origem da matéria-prima, até o produto final.

O artigo está organizando de acordo com as seguintes seções. A Seção II apresenta alguns trabalhos relevantes relacionados ao tema; A Seção III apresenta os principais conceitos sobre a temática abordada. A Seção IV apresenta a metodologia utilizada neste trabalho. A Seção V descreve a criação do aplicativo QRQueijo e o registro de queijos em Blockchain. Por fim, a Seção VI apresenta as conclusões do trabalho e indica possíveis estudos futuros

\section{Trabalhos Relacionados}

Esta seção apresenta alguns trabalhos relevantes, referentes a temática abordada neste trabalho, a respeito do uso de Blockchain para o registro seguro de informações.

Segundo Greve et al. (2018), a tecnologia Blockchain possui propriedades que garantem a segurança de seus dados, pois contém uma estrutura descentralizada e imutável. Os bancos de dados, embora possuam mecanismos de segurança, são suscetíveis a falhas por armazenarem os dados de forma centralizada, além de ser possível a sua alteração por indivíduos maliciosos. A Blockchain, diferentemente destes, realiza a validação dos dados e mantenha cópias exatas da cadeia de blocos em todos os nós.

Para Machado (2005), a rastreabilidade de alimentos é algo fundamental. Segundo o autor, a localização de uma entidade (ou item) por meio de identificações registradas, é uma forma de se utilizar a rastreabilidade de alimentos e assim gerar confiança na autenticidade dos produtos. Para que o consumidor de queijos obtenha informações relevantes, é necessário entender sobre a necessidade da rastreabilidade para a sociedade, onde a mesma acaba impactando na economia, no ambiente e também na produção dos alimentos.

De acordo com Resende e Lopes (2004), a rastreabilidade agrega fatores positivos para o gerenciamento, controle de riscos e garantia de qualidade de um produto. Em relação aos riscos, a rastreabilidade permite uma melhor tomada de decisão, com ações corretivas ou preventivas durante o processo de produção do alimento. Além disso, são ressaltados outros benefícios atrelados a cadeia produtiva (BRASIL, 2012), como o diagnóstico de problemas na produção e na imputação de responsabilidade; cumprimento da legislação, quando existente; agilidade e eficácia nos procedimentos de recall, protegendo a reputação da marca; dentre outras coisas.

No trabalho desenvolvido por Mendonça et al. (2020), é apresentada uma arquitetura para rastrear a cadeia 
produtiva do leite, utilizando a tecnologia Blockchain para armazenar e validar os dados coletados nos mais diversos pontos de controle do produto. O objetivo da pesquisa é oferecer um maior nível de confiabilidade e transparência às transações. A solução apresenta viabilidade no contexto de rastreabilidade do leite, uma vez que a proposta busca atender as demandas dos produtores e apresenta baixo impacto aos serviços já existentes.

Behnke e Janssen (2020) apresentam em seu trabalho uma discussão a respeito do uso de Blockchain no processo de rastreabilidade de alimentos. De acordo com os autores a tecnologia permite a geração de massas de dados que podem ser uteis para otimizar as cadeias produtivas de diversos tipos de alimentos, incluindo o leite e seus derivados. É apresentado um estudo de caso sobre o uso de Blockchain para rastreabilidade na gestão de cadeia de produção de leite e queijos em uma empresa. Verificou-se que com a tecnologia Blockchain é possível rastrear todas as informações relacionadas ao produto, desde o início da cadeia produtiva até o consumidor final. A utilização de Blockchain permitiu que os participantes da cadeia produtiva insiram e compartilhem informações de maneira identificada e abertamente auditável, gerando assim confiança ao processo de rastreabilidade dos alimentos.

\section{Conceitos e Definições}

Esta seção apresenta os principais conceitos abordados neste trabalho. Explica brevemente o que é uma rede Blockchain, como se dá o seu funcionamento e como a sua estrutura é organizada.

\subsection{Blockchain}

A tecnologia Blockchain surgiu em 2008 por meio de um artigo, com o principal intuito de proporcionar segurança e confiabilidade nas negociações financeiras que ocorriam na Internet (NAKAMOTO, 2008), sem que houvesse a necessidade de uma entidade central reguladora que controlasse as transações. No entanto, logo foi possível perceber que a cadeia de blocos poderia ser utilizada para o registro de uma série de outras informações, de forma segura e descentralizada (FERREIRA; PINTO; SANTOS, 2017). Desta forma, começou a surgir o interesse em seu uso em diversas outras áreas, dentre elas na rastreabilidade de alimentos.

Nakamoto (2008) descreveu em seu artigo sobre o Bitcoin, a primeira moeda digital a permitir transações financeiras sem intermediários, portanto descentralizada. O termo criptomoeda foi descrito pela primeira vez em 1998, por Dai, em seu trabalho sobre o "Dinheiro B", que é um sistema eletrônico e anônimo de pagamentos (DAI, 1998). A possibilidade de transações sem um intermediário é feita por meio de um sistema de registros distribuído, na qual são gravadas e verificadas por todos os usuários (nodos) da rede, definida como Blockchain. Esta tecnologia torna desnecessário o controle por uma entidade administradora, impossibilitando a manipulação nos valores e emissões das moedas.

Segundo Christidis e Devetsikiotis (2016), Blockchain é uma estrutura de dados distribuída, que é replicada e compartilhada entre os membros de uma rede. Esta estruturação permite que cada um dos nodos da rede (os chamados mineradores) avaliem as transações, validando e adicionando informações no banco de dados distribuído, chamado livro-razão

\subsection{Transações}

$\mathrm{Na}$ Blockchain as transações possuem endereços de entrada e saída. Os endereços de entrada correspondem àqueles que iniciam a transação, ou realizam o envio de valores. Já os endereços de saída informam o destino que irá receber tais valores ou mensagens. Em outras palavras, a tecnologia Blockchain funciona como um "livro razão", onde são armazenadas todas as transações que ocorrem entre os usuários da rede. Cada bloco possui uma assinatura digital, chamada hash, que serve como identificado único e inequívoco do bloco dentro da cadeia. Em cada bloco é armazenada uma referência para o seu anterior, o que forma assim uma cadeia de blocos, denominada Blockchain.

Antes de ser inserida no livro razão, cada nova transação deverá ser validada para garantir a integridade da rede. Está forma de validação, popularmente conhecida como mineração, realizada por todos os blocos da cadeia através de uma prova de trabalho (PoW, na sigla em inglês), que se utilizam do poder de processamento computacional e, consequentemente, gera um grande gasto de energia para solucionar cálculos matemáticos (CHICARINO et al., 2017).

Todos os computadores conectados à rede Blockchain guardam uma cópia da base de dados, onde se encontram o registro de todas as transações. Após um novo bloco ser validado e inserido na rede, todos os demais são atualizados para receber os novos valores e assim, garantir a integridade da Blockchain. A figura 1, apresentada abaixo, demonstra uma estrutura das transações na rede Blockchain. 


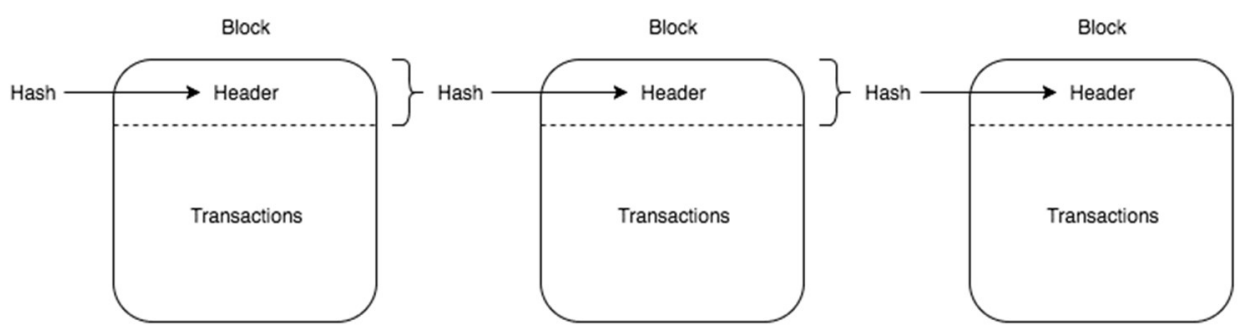

Figura 1 - Estrutura de transações da rede Blockchain.

Fonte: Liu (2019).

\section{Metodologia}

A classificação metodológica dessa pesquisa foi definida da seguinte forma (PRODANOV, 2013): Este trabalho caracteriza-se como uma pesquisa aplicada, tem como objetivo gerar novos conhecimentos para aplicação de caráter prático, dirigidos à solução de problemas específicos.

O método científico para a realização deste trabalho é definido como hipotético-dedutivo, onde a partir de um problema, formulam-se hipóteses, e com base nelas, entende-se as consequências que serão testadas ou refutadas.

Para uma melhor clareza sobre o problema e consequentemente formular as hipóteses sobre o mesmo, os objetivos de estudos deste trabalho foram classificados como exploratórios. Sendo assim, a abordagem foi definida como qualitativa.

O mecanismo técnico utilizado foi a pesquisa bibliográfica, que permitiu identificar informações em conteúdos já publicados e em materiais online, para análise posterior desses resultados

A execução deste trabalho foi dada da seguinte forma:

1. Definição do tema de estudo;

2. Definição dos objetivos;

3. Revisão de literatura sobre segurança da informação; Blockchain e sua aplicabilidade na rastreabilidade de produtos alimentícios;

4. Modelagem e implementação da aplicação baseada em Blockchain para registro das informações dos queijos;

\section{Resultados}

Esta seção descreve a implementação do aplicativo QRQueijo, apresenta o seu funcionamento e descreve o registro dos queijos em uma Blockchain local. Para a implementação foram utilizadas as seguintes ferramentas de desenvolvimento:

- Ruby on Rails: linguagem de desenvolvimento, que promove velocidade e uma boa escalabilidade;

- HTML5/CSS3: utilizadas para promover uma boa experiência de usabilidade aos usuários;

- MySQL: utilizada como base de dados, por sua popularidade e facilidade de uso;

- Docker: foi escolhida por permitir a criação de ambientes virtuais que podem instanciar serviços de forma eficiente, simulando o comportamento de até mesmo sistemas operacionais inteiros. E, para a rede Blockchain local, essa ferramenta permitirá a criação de nós para a simulação da rede;

- GIT: utilizada para o controle de versões da aplicação e repositório remoto na plataforma GitHub;

- Visual Studio: editor de texto que suporta uma grande quantidade de linguagens de programação;

- Android Studio: utilizado no desenvolvimento da aplicação Android.

\subsection{Sistema de registro}

Para ter acesso a aplicação faz-se necessário informar e-mail e senha, ou criar uma nova conta, caso o usuário ainda não a possua (Figura 2). Para garantir a segurança no momento do login, são realizadas validações por meio de um mecanismo de autenticação disponibilizado pelo Ruby on Rails, chamado Warden. Caso o usuário tente fazer qualquer autenticação que não esteja de acordo com as regras da aplicação, como: campos em branco, e-mail e senha inválidos ou também se o usuário não possuir cadastro na aplicação, é exibida uma mensagem de erro. 


\section{8xig}

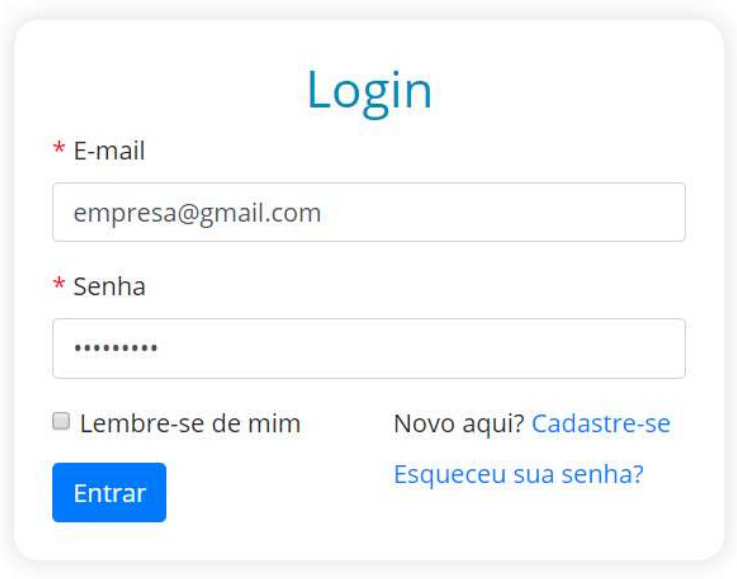

Figura 2 - Tela de login da aplicação.

Fonte: Desenvolvido pelo autor (2020).

Ao realizar o login com sucesso, o usuário é redirecionado para a página principal, onde é possível observar as principais funcionalidades da aplicação, como: visualizar a rede Blockchain, cadastrar um novo queijo, obter detalhes sobre um queijo já registrado, ou sair da aplicação. A Figura 3, apresentada abaixo, mostra a tela inicial da aplicação, bem como as suas principais funcionalidades.

QR Queijo Rede Blockchain Novo Queijo

\section{Lista de Queijos}

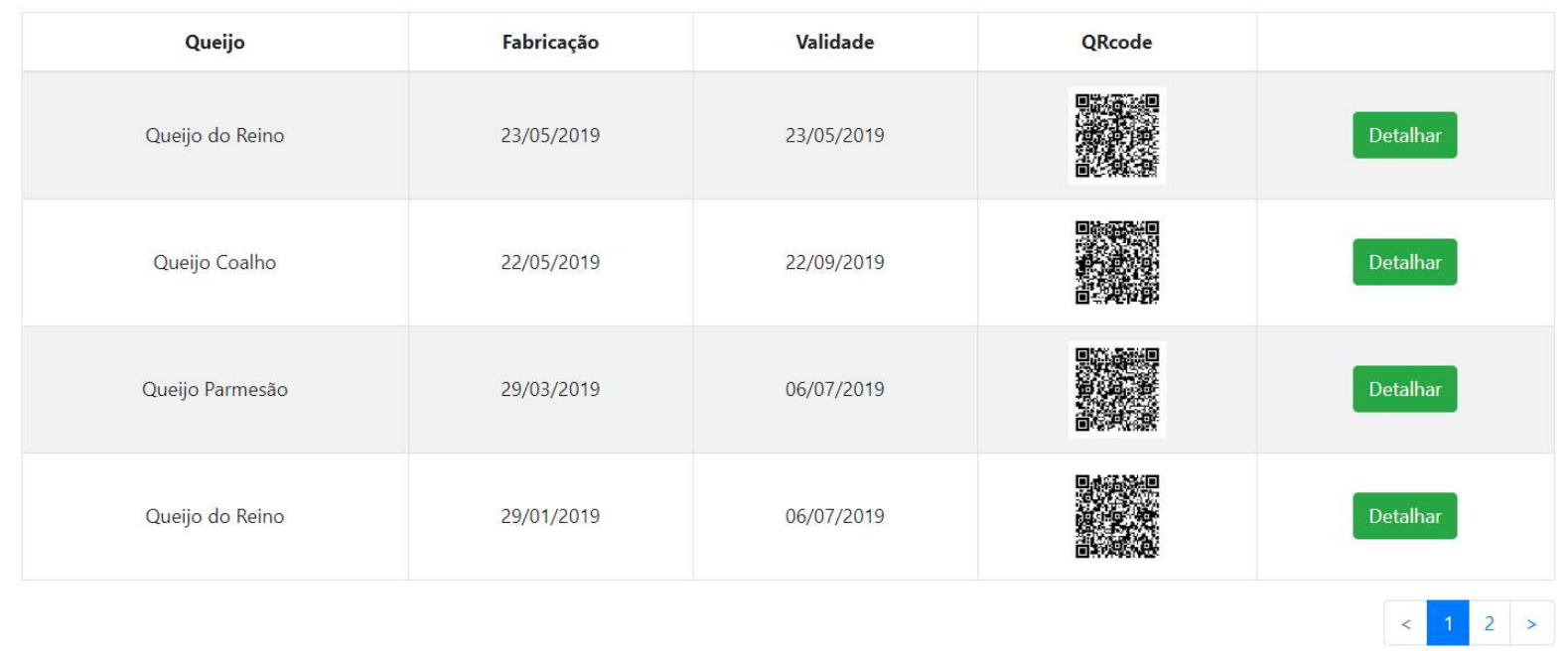

Figura 3 - Tela inicial da aplicação.

Fonte: Desenvolvido pelo autor (2020).

O sistema foi desenvolvido visando a sua aplicabilidade em um ambiente real, assim uma das suas funcionalidades básicas é o cadastro de novos queijos, que pode ser feito através de um botão na parte superior da tela, intitulado "Novo Queijo". Na tela de cadastro, apresentada na Figura 4, é possível informar os principais dados do queijo, como nome do fabricante, data de fabricação e validade, tipo do produto, empresa responsável e local de origem. 


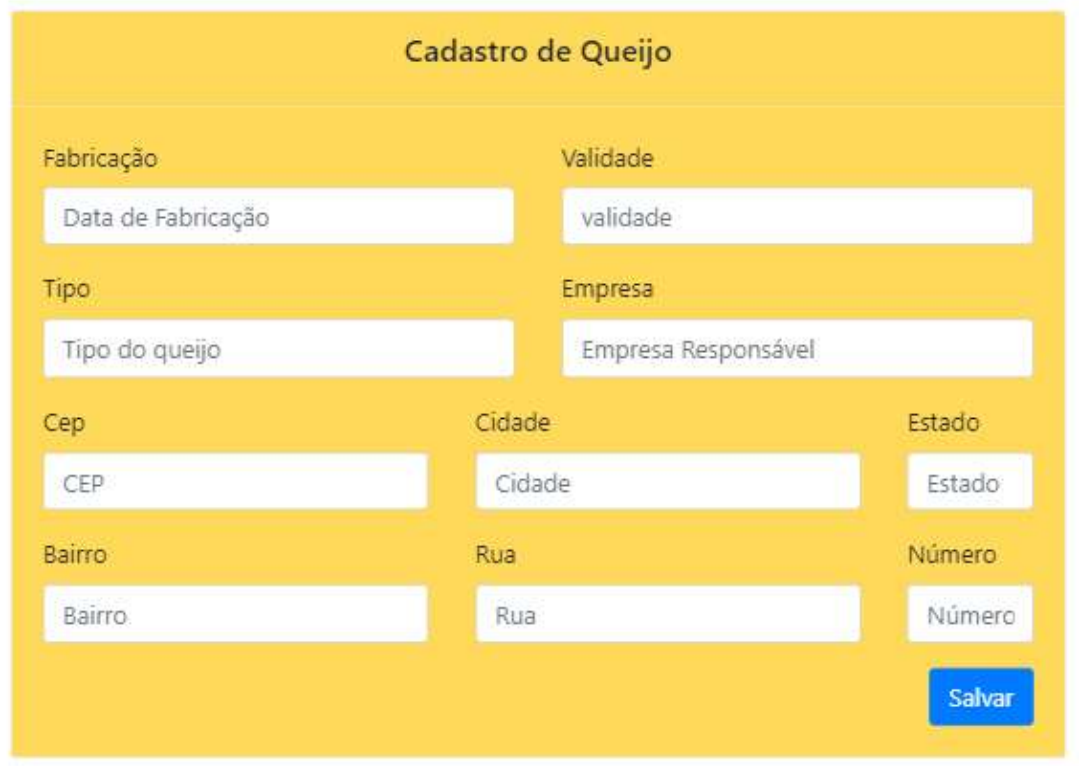

Figura 4 - Tela de cadastro de um novo queijo.

Fonte: Desenvolvido pelo autor (2020).

Ao clicar em salvar, é gerado um novo bloco no sistema contendo as informações, que serão validadas na Blockchain. Ao gravar um novo queijo, o sistema gera uma etiqueta contento um QRcode ${ }^{1}$, que será impressa e afixada na embalagem do produto. Na tela principal é possível visualizar detalhes dos queijos cadastrados (Figura 5), como informações do bloco onde ele foi gravado e a imagem do QRcode, que contém suas informações de rastreamento.

\section{Queijo do Reino}

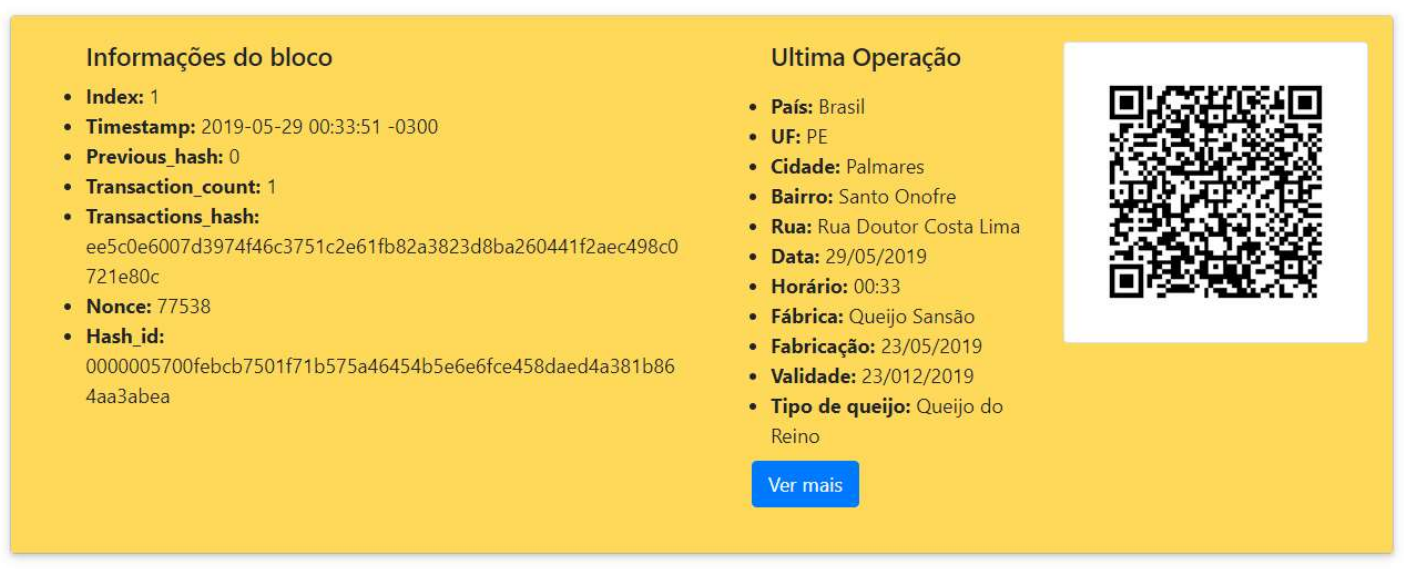

Figura 5 - Tela de detalhe de um queijo registrado.

Fonte: Desenvolvido pelo autor (2020).

\subsection{Aplicativo móvel QRQueijo}

Os usuários terão à sua disposição o aplicativo QRQueijo, que permitirá a realização da leitura do código QR e visualizar as principais informações de um queijo cadastrado. Para que isso seja possível, o produto deverá conter um identificador (QRcode) impresso em sua embalagem. A Figura 6, mostrada abaixo, apresenta a tela inicial do aplicativo QRQueijo, e apresenta a opção "Escanear Código", que permite ao usuário realizar uma nova leitura de dados de um produto.

\footnotetext{
${ }^{1}$ Do inglês Quick Response, código de barras bidimensional
} 


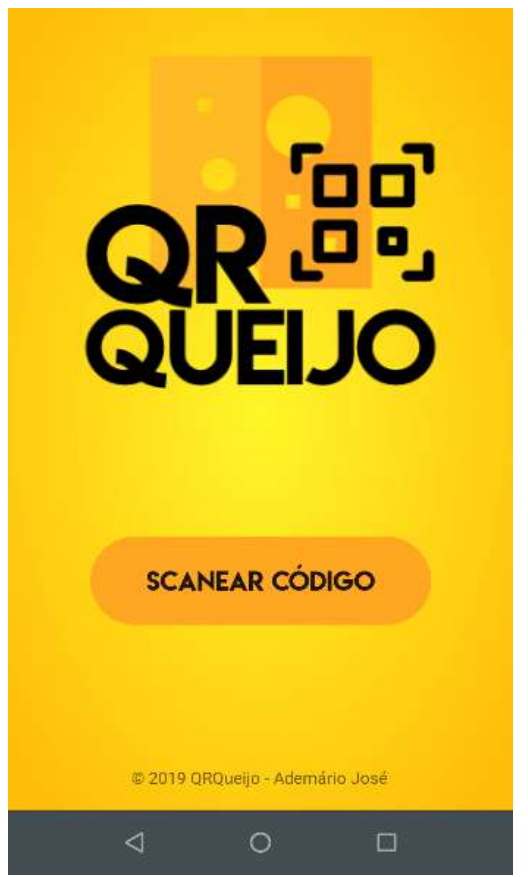

Figura 6 - Tela inicial do aplicativo QRQueijo.

Fonte: Desenvolvido pelo autor (2020).

O aplicativo além de realizar a leitura dos dados do produto, também realiza a atualização da localização do queijo registrado. Para cada nova leitura de um QRcode na embalagem de um queijo, um novo bloco será gerado na Blockchain contendo as informações do queijo e a sua nova localização, que é obtida por meio da permissão do usuário para a utilização da localização GPS ${ }^{2}$ do dispositivo móvel em que o aplicativo QRQueijo está instalado. A Figura 7 mostra um exemplo das informações de rastreamento de um produto.

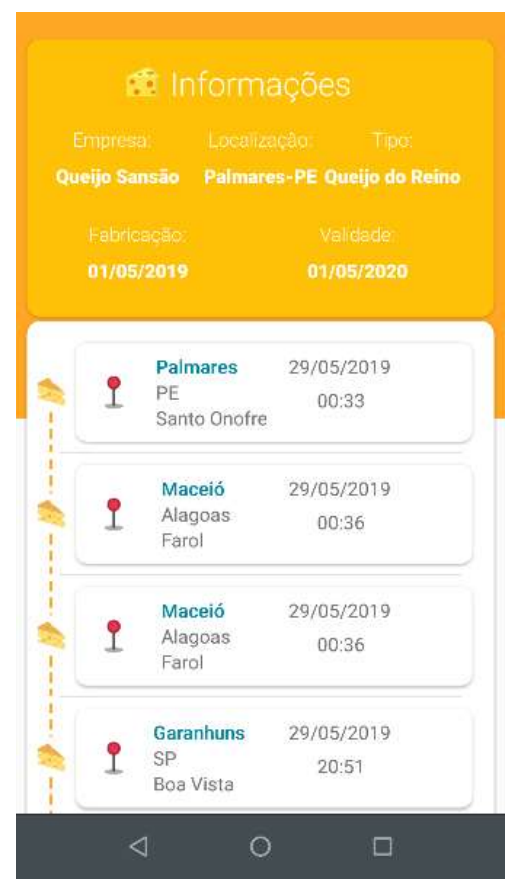

Figura 7 - Tela de rastreamento de um queijo cadastrado.

Fonte: Desenvolvido pelo autor (2020).

\footnotetext{
${ }^{2}$ Do inglês Global Positioning System
} 


\subsection{Registro na Blockchain}

Para registrar os dados dos produtos, realizou-se a implementação de uma rede Blockchain local. Para isso utilizouse como base a aplicação desenvolvida por Hartikka (2017) desenvolvida em Node.js. Após cada novo cadastro ou alteração nos produtos, os dados são armazenados no banco de dados da aplicação e simultaneamente enviados para a Blockchain, isso garante que a informação esteja disponível em dois locais distintos, e assim possa ser validada posteriormente, caso se faça necessário.

Para este trabalho foi instanciada uma cadeia de blocos contendo 10 nós, fazendo o uso de containers Docker para simulação dos nós da Blockchain, por ser essa uma tecnologia de alta performance e escalabilidade (MORAIS; CALLOU; LINS 2020). Todos os nós da rede permanecem em constante monitoramento, aguardando a ocorrência de alterações nos registros. Um novo bloco, ao ser gerado, deverá ser validado para então ser inserido à rede e atualizado em todos os nós.

Através da aplicação os usuários podem obter uma visualização completa dos registros que se encontram na rede Blockchain. Na Figura 8 mostrada abaixo, é possível visualizar detalhadamente as informações de cada um dos blocos, onde estão os registros dos queijos, sua data de fabricação, validade, o código QR de cada produto, como também a opção “detalhar”, que exibirá cada registro individualmente.

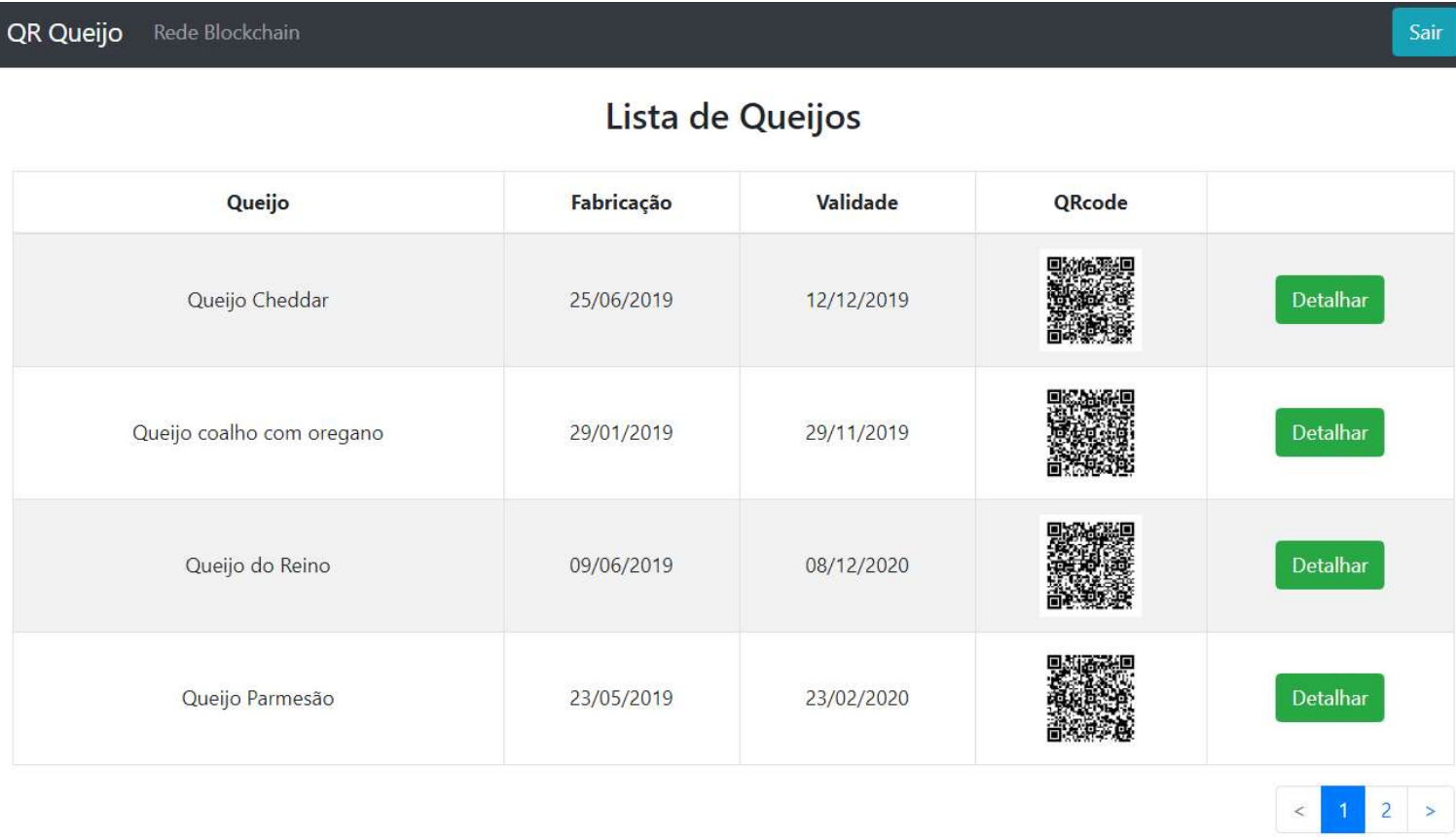

Figura 8 - Tela principal do administrador.

Fonte: Desenvolvido pelo autor (2020).

Ao cadastrar um novo queijo, a aplicação realiza a validação das informações automaticamente e registra na base de dados local. É realizada também, uma comunicação via HTTP com a rede Blockchain, sendo enviando o $h a s h^{3}$ das informações no corpo da requisição. Os demais nós realizamos as validações necessárias para garantir a integridade da rede, e então atualizam a Blockchain. A aplicação desenvolvida possui grande potencial de benefício a população e aos próprios fornecedores, pois garante informações seguras e atualizadas sobre os queijos produzidos.

A figura 9, apresentada abaixo, mostra o momento em que um novo registro é realizado na Blockchain, a linha amarela, no canto superior da imagem, destaca o valor do hash inserido, que se trata do identificador do queijo cadastrado. As linhas amarelas pontilhadas mostram o mesmo valor de hash sendo inserido nos demais nós da cadeia de blocos.

\footnotetext{
${ }^{3}$ Código criptográfico que representa os dados gravados
} 


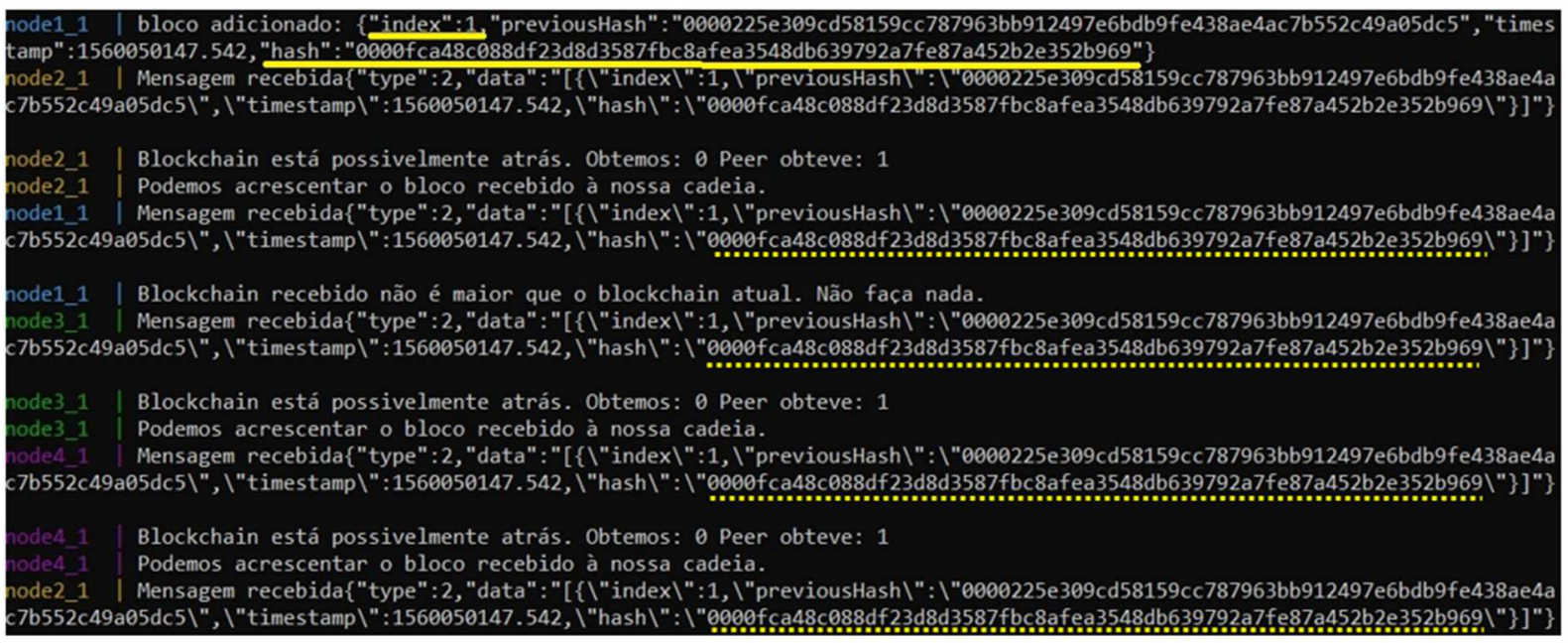

Figura 9 - Inserção de um novo bloco na Blockchain.

Fonte: Desenvolvido pelo autor (2020).

\section{Conclusões}

Este trabalho desenvolveu uma solução baseada em Blockchain que garante integridade e autenticidade as informações geradas por fabricantes de queijos, e permite a disponibilidade de tais informações aos consumidores.

Para ser possível chegar aos resultados apresentados, foi realizada uma revisão bibliográfica sobre as temáticas abordadas. Diante de casos reincidentes de fraudes e desinformações sobre queijos no Brasil, notou-se a existência de falhas de segurança nas informações como: identificadores de queijos e suas embalagens que são facilmente violadas e falsificadas; validades adulteradas; e também a falta de um registro em um órgão fiscalizador. Além disso, buscou-se conhecer os princípios de funcionamento e segurança de uma Blockchain. Em seguida foi realizada a implementação do sistema, visando garantir a integridade e a disponibilidade das informações através do registro imutável na cadeia de blocos.

Por fim desenvolveu-se o aplicativo QRQueijo, que permite rastrear toda a trajetória do queijo desde a sua produção até o consumidor final, garantindo assim a sua originalidade. A identificação por meio do QRcode, permite que esses produtos possuam uma assinatura digital, que tem como função selar o seu conteúdo, garantindo assim sua integridade e confiabilidade.

Como trabalhos futuros é desejável a realização de testes de segurança e disponibilidade da aplicação, para analisar a sua integridade e resistência a ataques. Também é possível melhorar a localização via GPS, para proporcionar uma melhor experiência ao usuário, como também outras formas de leitura dos dados para o QRcode. Pode-se realizar a integração do aplicativo com redes Blockchain pública, com a finalidade de garantir mais uma camada de segurança ao sistema de registros e geração de identificadores.

\section{Referências}

Behnke, K., \& Janssen, M. F. W. H. A. (2020). Boundary conditions for traceability in food supply chains using blockchain technology. International Journal of Information Management, 52, 101969.

Brasil. Rastreabilidade e segurança alimentar. Boletim Técnico-n. o , v. 91, p. 1-25, 2012.

Chicarino, V. R., Jesus, E. F., Albuquerque, C. V. N., \& Aragão Rocha, A. A. (2017). Uso de Blockchain para privacidade e segurança em internet das coisas. Livro de Minicursos do VII Simpósio Brasileiro de Segurança da Informação e de Sistemas Computacionais. Brasília: SBC, 28.

Christidis, K., \& Devetsikiotis, M. (2016). Blockchains and smart contracts for the internet of things. Ieee Access, 4, 2292-2303.

Costello, K. \& Omale, G. Gartner says global it spending to reach \$3.8 trillion in 2019. 2019. Disponível em: https://www.gartner.com/en/newsroom/press-releases/2019-01-28-gartner-says-global-it-pending-to-reach-3-8-trillio. Acesso em: 17/08/2019.

Dai, Wei. B-money, 1998. Disponível em: http://www. weidai. com/bmoney. txt. Acesso em: 08/04/2019. 
Ferreira, J. E., Pinto, F. G. C., \& dos Santos, S. C. (2017). Estudo de mapeamento sistemático sobre as tendências e desafios do Blockchain. GESTÃO. Org, 15(6), 108-117.

Greve, F. G., Sampaio, L. S., Abijaude, J. A., Coutinho, A. C., Valcy, Í. V., \& Queiroz, S. Q. (2018). Blockchain e a Revolução do Consenso sob Demanda. Simpósio Brasileiro de Redes de Computadores e Sistemas Distribuídos (SBRC)-Minicursos.

Hartikka, L. (2017) A Blockchain in 200 lines of code. Disponível em: https://medium.com/@lhartikk/ablockchain-in-200-lines-of-code-963cc1cc0e54. Acesso em: 06/09/2019.

Machado, R. T. M. (2005). Sinais de qualidade e rastreabilidade de alimentos: uma visão sistêmica. Organizações Rurais e Agroindustriais/Rural and Agro-Industrial Organizations, 7(1511-2016-131188), 227-237.

Mendonça, R. D., Gomes, O. S., Pereira, P. C., Vieira, A. B., \& Nacif, J. A. (2020, December). Utilização de Blockchain na Rastreabilidade da Cadeia Produtiva do Leite. In Anais do III Workshop em Blockchain: Teoria, Tecnologia e Aplicações (pp. 55-60). SBC.

Mol, J. (2015) Mais de 13 mil queijos da Canastra são confiscados pela Polícia Federal. Disponivel em: https://paladar.estadao.com.br/noticias/comida,mais-de-13-mil-queijos-da-canastra-sao-confiscados-pelapolicia-federal,10000011587. Acesso em: 13/09/2019.

Morais, A. M., Callou, G. R. A, \& Lins, F. A. A. (2020). Simulação e Avaliação de Desempenho de uma Rede Blockchain Utilizando Containers Docker. Cadernos do IME-Série Informática, 44, 73-87.

Moresi, E. A. D. (2000). Delineando o valor do sistema de informação de uma organização. Ciência da informação, 29(1), 14-24.

Mougayar, W. (2018). Blockchain para negócios: promessa, prática e aplicação da nova tecnologia da internet. Alta Books Editora.

Nakamoto, S; Bitcoin. A peer-to-peer electronic cash system. 2008. Disponível em https://bitcoin.org/bitcoin.pdf. Acesso em 13/09/2019.

Prodanov, C. C., \& de Freitas, E. C. (2013). Metodologia do trabalho científico: métodos e técnicas da pesquisa e do trabalho acadêmico- $2^{a}$ Edição. Editora Feevale.

Resende, E. H. S., \& LOPES, M. A. (2004). Identificação, certificação e rastreabilidade na cadeia da carne bovina e bubalina no Brasil. Lavras: UFLA, 38.

Scarfone, K., Jansen, W., \& Tracy, M. (2008). Guide to general server security. NIST Special Publication, 800(s $123)$.

Tanenbaum, A. S., \& Steen, M. V. (2007). Sistemas Distribuidos: principios e paradigmas. 